\title{
Notes sur le $\mathrm{XII}^{e}$ Congrès International de Laiterie
}

\author{
Stockholm, du 15 au 19 Ẻô̂t 1949
}

\author{
par le Dr L.-M. FEUNTEUN
}

Délégué du Ministère de la France d'outra-mer

L $E_{X} X I I$ e Congrès International de Laiterie qui s'est tenu à Stockholm, du 15 au 19 Août 1949, a été ouvert par S.A.R. le Prince Royal Gustav Adolf et présidé par le Professeur Docteur R. Burri, Président de la Fédération Internationale de Laiterie, représentant de la Suisse.

Le Congrès, qui a siégé au Palais du Parlement, a groupé 2.000 congressistes venus de 59 pays.

Le matériel adressé au Congrès sous la forme de rapports était ample, lourd, hétérogène; son étude n'était pas simple et les difficultés de synthèse se retrouvent parfois dans quelques imperfections ou erreurs de certains rapports généraux.

Le travail effectué dans les diverses sections aboutit à des propositions de résolutions qui furent présentées à la séance pleinière de clôture. Les travaux du Congrès étaient classés en six sections :

\footnotetext{
$\mathrm{I}_{r}$ - Production du lait, hygiène et contrôle.

II. - Physique, chimie et microbiologie.

III. - Côté technique du problème.

IV. - Économie et commerce.

V. - Organisation de l'industrie laitière.

VI. - Laiterie tropicale.
}

Nous donnerons sous forme de notes successives un aperçu des travaux des cinq premières sections et traiterons à part, et plus longuement, ceux de la section Laiterie tropicale.

\section{I. - PRODUCTION DU LATT, HYGIÈNE ET CONTROLE}

L'action des fourrages courants dans la production laiterie est maintenant bien connue. Théoriquement, on peut par leur emploi influencer la qualité du lait dans le sens désiré; cependant, dans la pratique, on se heurte souvent à de grandes difficultés et, parfois, à d'insurmontables obstacles car la production de lait de qualité coûte cher et on ne dispose pas toujours du fourrage voulu.
Au sujet de la teneur du lait en vitamines, on constate que cette teneur s'abaisse en hiver mais qu'on peut l'élever par l'emploi de fourrages appropriés, malheureusement coûteux, ou par l'addition de vitamines synthétiques. Ce dernier procédé est en usage en Amérique.

L'administration de fourrages ensilés en quantité suffisante permet de maintenir dans le lait une teneur élevée en vitamines, mais a l'inconvénient, par suite de la présence de bactéries butyriques, de rendre le lait impropre à la production de certains fromages.

On a tenté d'augmenter la production laitière par l'usage d'hormones galactogènes.

Le paiement de la qualité pose de difficiles problèmes : dans les pays de grande productión de beurre, on paie le lait suivant la teneur en matiere grasse, il faudrait également arriver à payer la qualité suivant la teneur en protéine, pour' les laits destinés à la production de fromages; encore faudrait-il mettre au point une méthode simple pour déterminer la teneur en protéines.

Il est démontré que la teneur en protéines du lait, aussi bien que sa teneur en matière grasse, constitue un caractère héréditaire chez les femelles laitières. Une sélection sur cette base est possible.

L'industrie laitière a une grande tâche à remplir dans le domaine du traitement des maladies du bétail laitier : tuberculose, brucellose, mammite. Cette tâche consiste principalement dans l'éducation et la propagande, le contrôle et la notification des cas de maladie, la pasteurisation du lait écrémé et du petit lait, la différenciation des prix, enfin, l'assistance technique et économique dans la lutte contre les maladies.

Dans la plupart des pays, l'État a pris en main le contrôle des maladies, mais compte sur l'assistance financière de l'industrie laitière.

Le Congrès a discuté l'adoption de méthodes simples pour le diagnostic des maladies des vaches laitières. 


\section{II. - PHYSIQUE, CHIMIE ET MICROBIOLOGIE}

Par ultra-centrifugation, on est arrivé a fractionner la caséine en éléments de grandeur moléculaire et de compositions différentes. La matière grasse du lait se trouve sous forme de gouttelettes dont la structure peut être étudiée au microscope électronique. Cette structure influence la consistance des produits laitiers. Les consommateurs estiment la consistance du beurre ou du fromage bonne ou mauvaise, mais il appartient aux chercheurs d'améliorer la consistance par des méthodes exactes de mesure. La science. nouvellé traitant de ces ćtudcs est la rhéologiè.

Dans la composition chimique des produits laitiers, les substances qui sortent de l'ordinaire, mais qui ont une action speciale sur l'organisme, attirent le plus l'attention.

La matière grasse du lait contient un facteur de croissance sur la nature duquel on n'est pas encore fixé.

Les substances conférant aux fromages une saveur spécifique et les méthodes utilisées pour produire les quantités optima de ces substances posent un intéressant problème.

L'importance de la formation des amino-acides et substances analogues lors de la maturation des fromages a été démontrée.

Une importante découverte pratique concernant l'action de l'humidité sur la conservation de lá poudre de lait a été faite. Il a été démontré que les groupes aminés libres de la protéine réagissent avec le lactose et influencent la solubilité, la couleur et la valeur alimentaire de la poudre.

Il est intéressant de noter qu'en Nouvelle-Zélande la composition de la matière grasse du lait, déterminée par l'index iode, varie suivant la saison et la végétation d'une manière très différente de ce qui est obser vé dans l'hémisphı̀re nord.

Dans la chimie du lait, l'oxydation, et notamment l'oxydation des graisses, occupe une place toute particulière.

Les rapports adressés au Congrès donnent une bonne vue d'ensemble des facteurs qui provoquent l'apparition des défauts de saveur. On sait que ces défauts viennent de la décomposition de certains acides gras; un nouvel acide plus sensible que les autres a été découvert. La quantité de cet acide présent dans le beurre varie suivant l'alimentation et il existe une relation entre cette quantité et les défauts de saveur. Des antioxydants, destinés à empêcher l'apparition de ces défauts, ont été essayés. La plus récente découverte dans ce domaine est un antioxydant préparé à partir du petit lait.

Les bactériophages ont causé bon nombre de difficultés dans la fabrication dı fromage quand on a utilisé dans cette fabrication des bactéries lactiques pour acidifier le lait : après étude du problème, des mesures préventives ont été prises, mais certaines souches de bactéries lactiques constituent des antibiotiques gênants dans la fabrication du fromage.

Une substance ayant une action antibiotique générale, et qui peut être utilisée contre la tuberculose et la mammite, a été isolée.

\section{III. - PROBLÈMES TECHNIQUES}

L'opportunité de la pasteurisation dú lait de consommation est admise. Pasteurisation et réfrigération ont permis de réaliser un accroissement de la consommation, la valeur nutritive du lait ne souffre pas de la destruction des agents pathogènes.

Il est surprenant de constater que les rapports adressés au Congrès n'apportent que peu d'éléments nouveaux au sujet des méthodes de pasteurisation, même en ce qui concerne la méthode' de pasteurisation par la haute fréquence.

Ce fait laisse suppoșer que les nouvelles méthodes ne sont pas prêtes à affronter la critique. Toutefois, on constate une tendance générale à réduire le temps de pasteurisation.

L'opportunité de la pasteurisation du'lait destiné à la fabrication de produits laitiers est discutée. Dans certains pays tout le lait est pasteurisé; dans d'autres, on estime le procédé contre-indiqué pour les laits destinés à la production du fromage.

Aux États-Unis, on se sert d'un nouveau pasteurisateur, le "Vacreator ». Il pasteurise le lait dans le vide en trois temps et présente des avantages tant pour la fabrication du beurre que pour la préparation de la poudre de lait.

Le contrôle de l'efficacité de la pasteurisation cst très important.

Dans les rapports au Congrès, on trouve la descriplion de plusieurs méltıodes s'y rapportant et qui permettent aux laiteries un contrôle rigoureux des laits qu'elles traitent.

L'homogénéisation du lait de consommation n'a pas encore atteint le degré de diffusion de la pasteurisation et n'a pas, du reste, la même importance fondamentale.

Aux États-Unis, on homogénéise la plupart des laits de consommation. On obtient ainsi un lait plus savoureux et on évite aussi les « bouchons'》 de crème dans les bouteilles de lait.

Il y a dix ans qu'a été lançée l'idée d'une technique nouvelle de fabrication du beurre selon laquelle la matière grasse du lait pourrait être transformée en beurre par un procédé continu. Cette technique a été mise au point et se trouve réalisée par quatre types d'appareils présentés au Congrès : du lait 
est versé à une extrémité de l'appareil, du beurre en sort de l'autre extrémité.

Les appareils fonctionnent bien dans la pratique.

Dans la fabrication du fromage, c'est le stockage par le froid qui retient le plus l'attention. C'est sans doute là une conséquence de la tendance générale de l'application du froid à la conservation des denrées alimentaires.

La crème glacée (ice cream) n'est plus un article de luxe, mais est maintenant considérée comme un aliment hautement nutritif. Les rapports au Congrès traitent de la fabrication, des méthodes de contrôle, des défauts des produits et des moyens d'y remédier.

Le lait en poudre ou lait séché gagne de plus en plus en importance, soit qu'il serve à stocker les surplus de lait, soit qu'il serve à approvisionner les régions dépourvues de lait frais. Techniques de préparation et de conservation, ainsi que qualité du lait en poudre, se sont perfectionnées.

Un grand nombre de rapports traitent du nettoyage et de la désinfection du matériel de laiterie, de la standardisation des pièces détachées, des problèmes de construction de laiterie et d'évacuation des eaux résiduaires.

\section{IV. - PROBLÈMES ÉCONOMIQUES}

II est surtout question de l'amélioration du rendement et de la réduction des frais. Il y a là une multitude de problèmes de détail; main-d'œuvre, transports aux laiteries, emballag'e, distribution, etc.

La valeur nutritive des produits laitiers, eu égard à la santé publique, constitue aussi un probleme important.

Les possibilités d'extension du marché du lait sont aussi étudiées, cette extension est généralement liée au pouvoir d'achat des consommateurs.

\section{V: - SITUATION DE L'INDUSTRIE LAITIÈRE DANS DIFFÉRENTS PAYS}

On considère qu'il serait très intéressant d'avoir le maximum d'information sur l'organisation de l'industrie laitière dans tous les pays. Une trentaine de pays ont répondu à l'enquête effectuée au sujet de cette organisation.

Il est aussi question des mesures officielles prises dans différents pays pour développer la fabrication de produits laitiers.

\section{VI. - LAITERIE TROPICAEE}

Le Comité de cette section était composé comme suit :

Président : E. Lancelot (France).
Vices-Présidents : O..E. Reed (États-Unis), R.-J. Simmons (Grande-Bretagne).

\section{Membres :}

N.-K. Bhargawa (Inde). - N. Craig (lle Maurice). - R.-P. Delpech (Argentine). - M. Delrieu (Algérie). - C. Errazuruz (Chili). - A.-H. Fahmi (Égypte). L.-M. Feunteun (France d'outre-mer). - O. Frensel (Brésil). - L.-S. Harmsen (Indonésie). - E. Hegh (Belqique). - E.-R. Léon (Cuba). - P. Mahadevan (Ceylan). - C.-F. Neyzen (Congo belge). - S.-E. Piercy (Kenya). - F. Simpson (Gold Coast). M.-R. Soetisno (Indonésie). - H.-M. Stuchberry (Tanganyika). - E.-G. White (Grande-Bretagne).

Les questions traitées par cette section étaient les suivantes :

1. Production rationnelle du lait par l'introduction de races appropriées, amélioration des pâturages, etc.

2. Production de beurre. Fromage et autres dérivés du lait.

3. Le ravitaillement en lait des grandes villes.

Nous donnons ci-après, dans l'ordre de leur publication, une analyse des rapports classés dans cette section.

\section{Progrès du croisement avec le bétail d'origine indienne, par O.-E. Reed, Bureau of dairy Industry, Washington (U.S.A.).}

Des recherches sont en cours au Bureau de l'Industrie Laitière du Ministère de l'Agriculture des États-Unis, tendant à développer de nouvelles races laitières résistantes à la chaleur et qui puissent être adaptées aux diverses régions tropicales des ÉtatsUnis.

Des zébus de l'Inde (Brahman cattle) ont, depuis plus de cent ans, éte acclimatés aux États-Unis; on en trouve actuellement dans quarante états de l'Union. Les croisements Brahman et Shorthorn effectués au King Ranch à Kingsville, Texas, ont abouti à la création de la " race » Santa Gertrudis, renommée dans les États du Sud.

En 1946, on a importé aux U.S.A. deux vaches et deux taureaux de la race du Sind (Indes) qui ont été croisés avec la race Jersey. Une des vaches importées vient de terminer sa première lactation, donnant un rendement total de lait et un rendement de matière grasse supérieurs à ceux jusqu'ic observés sur le bétail de Louisiane.

Outre des recherches sur le lait et le beurre, des études sont faites sur la physiologie de ces animaux, leur alimentation, leur résistance aux maladies, etc.

Des expériences analogues, à partir du bétail Sind et Jersey, sont également effectuées à l'Institut d'Allahabad (Indes). 
Production de lait, introduction de races amélioratrices, alimentation du bétail dans les territoires' français sous climat tropical, par le Docteur L.-M. Feunteun; Chéf du Service de l'Élevage et des Industries animales, Ministère de la France 'd'outre-mer, Paris (France).

La production laitière des races bovines des territoires français d'outre-mer est extrêmement faible. Du point de vue qualitatif, les laits coloniaux se distinguent des laits européens par leur forte teneur en matières grasses, et surtout en matières albuminoïdes, qui les rend difficiles à utilișer dans l'alimentation des jeunes enfants.

Les laits de conserve ne pouvant cependant répondre à tous les, besoins, le Service de l'Élevage des Colonies a été amené à entreprendre l'amélioration de la production laitière, afin d'assurer aux centres urbains importants un approvisionnement convenable en lait frais; beurre et fromage.

Cependant, la protection du cheptel contre les épizooties ayant constitué pendant de nombreuses années le principal souci en matière d'élevage, ce n'est que tardivement que l'action zootechnique visant l'accroissement de la production laitière a pu être entreprise.

De nombreux essais de croisements avec des races importées ont été réalisés avec des résultats variables.

Dans certaines régions de Madagascar et du Cameroun, relativement favorisées sous le rapport 'de l'alimentation des animaux; les expériences ont été couronnées de succès. Par contre, en Afrique Occidentale et en Afrique Équatoriale, les expériences n'ont pu prendre tout le développement nécessaire, mais les essais réalisés comportent de précieux enseignements dont il sera possible de tirer parti dès qu'il sera possible d'améliorer le milieu, condition sine qua non de l'amélioration du bétail.

\section{Le mauvais rendement du bétail laitier dans les} régions tropicales, analyse des problèmes, par W.-C. Miller, Animal health trust New Market, Suffolk (Grande-Bretagne).

L'auteur discute l'intérêt d'une augmentation de la quantité de lait pour satisfaire aux besoins des populations des régions tropicales.

La plupart des efforts pour améliorer le bétail laitier de ces régions se sont soldés par des insuccès. Laugmentation sur certains points, de la production de lait, a généralement été dưe à un accroissement du nombre de vaches mises en service et non à une amélioration du rendement individuel.

M. Miller souligne la mauvaise qualité des races indigènes des pays tropicaux et la nécessité de reconsidérer les problèmes d'élevage dans ces pays.

II note également la nécessité d'appuyer et de compléter les méthodes génétiques par une meilleure: alimentation ainsi que par des mesures visant à une mellleure exploitation des troupeaux laitiers.

La défense contre les maladies et l'assistance aux éleveurs en cas de perte sont considérées.

Selon l'auteur, des techniciens européens ainsi qu'une amélioration de l'enseignement chez les autochtones sont nécessaires.

\section{Amélioration du bien-être et de l'alimentation des vaches pendant les mois d'été, par D.-M. Seath, Dairy Section College of Ágricul- ture, Lexington, Kentucky (U.S.A.):}

Six expériences sur le comportement des, vaches pendant les mois d'été ont été effectuées 'par l'auteur et ses collaborateurs.

Les expériences faites à Bâton-Rouge (Louisiane) ont montré que des vaches Jersey et Holstein en lactation passent la plus grande partie de la journée à l'ombre, pendant les journées claires et chaudes (température moyenne $29^{\circ} 7$ C.). Le temps de pâture diurne n'excédait pas en moyenne une heure trois quarts; le temps de pâture nocturne était plus de trois fois plus long. Pendant les journées relativement fraîches, le temps de pâture de jour était de 2,4 fois plus long et le temps total de pâture sur vingtquatre heures était augmenté de plus d'une heure.

Trois expériences furent effectuées dans le but de déterminer l'efficacité de diverses méthodes pour rafraîchir les vaches pendant le's mois d'été. Le mouvement dé l'air produit par un ventilateur dirigé sur les vaches à l'ombre sous un abri s'est révélé plus efficace que l'ombre seule. L'aspersion des vaches avec de l'eau à $29^{\circ} \mathrm{C}$. les rafraîchit plus vite que ne le fait le ventilateur, mais au bout d'une heure la différence est peu considérable.'

L'aspersion, combinée avec l'action d'un ventilateur, est plus efficace quie chacune des deux autres méthodes utilisées seules.

Un dispositif d'aspersion par eau sous pression. produisant un brouillard très fin que les vaches appréciaient et utilisaient spontanément pendant les journées chaudes, fut essayé. Il a été constaté, avec ce système de douche, que la température du corps des vaches soumises à l'expérience resta normale ou diminua même et que le rythme de' la' respiration se maintient voisin de la normale.

A Bâtọn-Rouge (Louisiane, U.S.A.) en 1947, et à Lexington (Kentucky, U.S.A.) en 1948, des essais ont été faits dans le but de maintenir la production laitière à un niveau élevé par distribution dẹ foin aux vaches en plus de la pâture. Ni lés variations. de production du latt, ni les variations de poids des animaux, ne donnèrent d'indications statistiques significatives quant à l'influence de la distribution de foin. 
Le ravitaillement en lait au Congo belge, par L. Geurden (Belgique).

Ce rapport situe les premiers efforts effectués en vue de la production de lait au Congo belge et indique quelques-unes des difficultés rencontrées.

Des croisements du bétail autochtone avec les races Jersey, Friesland, Ayrshire, Siementhal et Devon ont éte tentés. Les résultats ne sont pas concluants, mais les meilleurs ont été obtenus avec la Friesland.

Actuellement. le lait de consommation est produit près des agglomérations. A Elisabethvilile, une Société Coopérative vend du lait pasteurisé en bouteilles.

Les facteurs concernant l'augmentation numérique, la protection sanitaire et le perfectionnement du bétail laitier sont étudiés.

\section{Amélioration de la production du lait et de la matière.grasse par l'organisation de l'insé- mination artificielle, par E.-J. Perry, Dépar- ternent de l'Industrie Laitière, New-Jersey (U.S.A.).}

Ce rapport ne traite pas spécialement de l'élevage en-milieu tropical. Perry signale que la pratique systématique de l'insémination artificielle est profitable si l'on tient comple de l'augmentation de production qui résulte de la supériorité des caractères héréditaires des mâles utilisés.

\section{Organisation et développement de l'insémi- nation artificielle du bétail laitier du Michigan, par A.-C. Baltzer, Michigan State Collège (U.S.A.).}

Cet exposé n'a que des rapports très indirects avec la laiterie tropicale; l'auteur décrit les méthodes utilisées par les autorités de Land Grant College (Michigan), dans l'établissement des coopératives d'élevage de bétail laitier de cet État, utilisant l'insémination artificielle (création d'un centre d'insémination, formation d'inséminateurs). En 1944 , quatre associations existaient pour 1.400 vaches, en 1949, le nombre d'associations était de cent trente-quatre pour 121.000 vaches.

Résultats de recherche sur la sélection du bétall laitier, par M.-H. Fohrman, Bureau of Dairy Industry, Washington (Ü.S.A.).

Ce rapport ne concerne qu' indirectement la laiterie tropicale, Forhman résume les résultats obtenus au cours d'expériences poursuivies au cours des trente dernières années par le Bureau de l'Industrie Laitière du Ministère de l'Agriculture des U.S.A.
Le rapport contient des statistiques et renseignements divers sur les accroissements de rendement résultant de l'emploi de l'insémination artificielle.

Le croisement des races diverses en vue de l'augmentation du rendement est préconisé. Le rendement domine l'élevage.

Taureaux éprouvés aux États-Unis, par J.-F. Hendrick, Bureau of Dairy Industry, Washington (U.S.A.).

Ce rapport n'a pas directement trait à la laiterie tropicale. L'auteur indique qu'un géniteur mâle éprouvé est un animal dont cinq filles prises au hasard ont eu leurs graphiques de production. comparés avec ceux de leurs mères en vue d'établir l'aptituide du mâle à transmettre ses caractèresłà sa descendance.

Ces études par graphiques ont été effectuées par les associations pour l'amélioration du bétail laitier aux U.S.A.

Depuis 1935. plus de 25.000 géniteurs ont été éprouvés.

La production moyenne de lait et de matière grasse des filles de ceś génitcurs est de 4.185 kilogrammes de lait et $166 \mathrm{~kg}$. 5 de beurre. La production moyenne de toutes les vaches traites aux ÉtatsUnis est de 2.250 kilogrammes de lait et de $89 \mathrm{~kg} .55$ de beurre.

1.700 géniteurs éprouvés sont actuellement utilisés pour l'insemmination artificielle.

\section{Les sociétés coopératives poux l'élevage arti- ficiel du bétail laitier aux États-Unis, par J.-F. Hendrick.}

Ce second rapport de Hendrick donne des détails sur l'organisation des Associations d'Élevage basées sur l'insémination artificielle aux États-Unis : administration, manipulation du sperme, frais d'exploitation.

\section{Utilisation du lait dans les conditions particulières} de l'Égypte, par A.-H. Fahmi, Département de laiterie Giza (Égypte).

L'utilisation du lait en Égypte est subordonnée aux conditions climatiques qui varient du nord au sud.

La production du lait, par rapport au cheptel et a la population humaine, est faible. La consommation, par tête, est de 12 kilogrammes environ. Cette situation est due aux faits suivants : l'agriculture passe avant l'élevage, le bétail laitier est mauvais, l'exploitation du bétail défectueuse et les possibilités d'achat des consommateurs, faible. 
Le lait produit est utilisé comme suit :

- $20 \%$ lait de consommation;

- $20 \%$ préparation de fromages;

- $60 \%$ préparation de beurre.

$70 \%$ du lait de consommation est du lait de bufflesse. Ce lait est généralement consominé après ébullition. La pasteurisation n'est pratiquée que dans quelques grandes villes.

La fabrication de laits fermentés diver's et d'ice cream est importante.

Les fromages sont préparés à l'aide de laits de vaches et de brebis; leur production est réduite par suite de l'insuffisance de la production de lait.

Ce rapport traite de différentes variétés de fro" mages et de la production de beurre et de samn (beurre déshydraté).

\section{Production de beurre, fromages et autres dérivés du lait en Äfrique française du Nord et dans les pays de I'Union française, par E. Lan- celot, École Nationale Supérieure des Industries du Lait, Paris.}

Le rapport traite de la production des beurres et fromages dans les pays français d'outre-mer placés sous un climat chaud : pays méditerranéens, subtropicaux, tropicaux et équatoriaux.

En Algérie et Tunisie, la production est inexistante; au contraire, l'appel de la consommation est important.

Au Maroc, un mouvement vers la beurrerie et la fromagerie se dessine très nettement en vue de l'approvisionnement de la population locale et de celle des pays voisins.

En Afrique Occidentale et Équatoriale, on constate une importante production de beurres fondus. Des perfectionnements peuvent être apportés aux techniques de fabrication employées pour obtenir des produits exportables.

A Madagascar, dans certaines régions de hauts plateaux, les 'réalisations actuelles autorisent des espoirs sérieux; au sujet du ravitaillement en lait et produits laitiers des villes.

En Nouvelle-Calédonie, le-climat permet également de créer une production fromagère et beurrière.

Dans tous les pays ci-dessus indiqués, le premier effort à accomplir consiste à améliorer et organiser' la production du lait par des mesures zootechniques et agronomiques appropriées.

Distribution du lait aux Indes, par N.-K. Bhargava, Provinces Unies (Indes).'

L'élevage bovin tient une place essentielle aux Indes. Le pays, possède un tiers du bétail bovin recensé dans le monde.
Climat, distances et difficultés de transport font que les effectifs de vaches laitières sont très élevés autour des grandes villes.

Les vaches sont généralement sous-alimentées et leurs rendements en lait sont faibles. Les mâles. sont utilisés pour le travail.

La plupart de la population étant végétarienne, le lait est souvent pour elle la seule source valable de protéine; malheureusement, la consommation est limitée par le faible pouvoir d'achat des classes pauvres.

Le problème de la production et de la répartition ne se pose que pour les grandes agglomérations.

Pour les grandes villes, on a créé des établissements laitiers qui prennent les vaches aprè vêllage, les gardent un an sans les, faire saillir, puis les vendent à la boucherie:

Les chemins de fer n'ont rien fait pour favoriser les transports de lait : transports chers, manque de wagons réfrigerés. Les transports par camions sont réduits par le manque de routes. Le mode de transport le plus généralement utilisé est la bicyclette.

Étant donné le manque d'hygiène a la production et d'outillage pour le transport, le lait doit être bouilli par le consommateur.

Le lait de bufflesse, plus riche en matiere grasse, est vendu plus cher que le lait de vache.

La pauvreté deg producteurs et le faible rendement de leurs vaches rendent très difficile l'amélioration de l'équipement et des méthodes de travail.

Le lait est distribué deux fois par jour et rapidement vendu quelles que soient sa qualité et ses altérations. La pasteurisation, introduite aux Indes par l'armée, n'est pas appliquée par plus d'une douzaine d'établissements laitiers pour tout le pays: Le fait que l'ébullition est pratique courante a retardé l'adoption de la pasteurisation.

Le perfectionnement du produit mis en vente est difficile du fait que l'acheteur ne veut pas payer la qualité et que les petits producteurs sont tenus par les revendeurs.

Un système rationnel de ramassage et de distribution est souhaitable:

La mise en application d'un contrôle sanitaire efficace est une nécessité urgente.

Une réorganisation complète de l'industrie laitière est nécessaire; elle doit commencer par l'élimination des villes du bétail laitier.

Des expériences récentes effectuées à Lucknow ont indiqué la voie à suivre en matière de commerce du lait : création de sociétés coopératives de production avec centres de ramassage dans les villages où les éleveurs amènent leurs vaches à traire. Le lait, correctement collecté, est transporté en 'camion à la laiterie de la ville où il est pasteurisé avant distribution. 
A ces mesures, doit s'ajouter le perfectionnement du bétail et de son alimentation.

Une réglementation devrait :

10 Intensifier la production de lait dans les villes;

$2^{\circ}$ Rendre obligatoire la licence pour les producteurs ruraux;

$3^{0}$ Imposer des conditions hygiéniques à la production. au ramassage et à la distribution;

$4^{\circ}$ Fixer les standards bactériologiques et chimiques du lait;

$5^{\circ}$ Fixer le prix du lait.

Ravitaillement en lait des centres urbains en Afrique française du Nord et dans les pays de l'Union française, par E. Lancelot, École Nationale Supérieure des Industries du Lait, Paris (France).

Le rapport décrit la situation actuelle du ravitaillement en lait des centres urbains des territoires français d'outre-mer. Il indique les possibilités d'amélioration de cette situation.

Les réalisations et les projets en cours au Maroc sont particulièrement remarquables et ce pays peut servir de pilote pour tous les autres.

En Algérie et Tunisie, où le problème se pose avec acuite pour Alger, Oran, Tunis, des projets sont en cours d'étude ou de réalisation, mais leur succès est'subordonné à la création de cultures fourragèes irriguées.

En Afrique noire et en Indochine, malgré quelques essais entrepris sous la pression du blocus pendant la dernière guerre, c'est le lait de conserve importé qui constitue la source principale d'approvisionnement.

A Madagascar, les ressources locales peuvent être être suffisantes pour ravitailler les centres importants de l'île.

Le rapport conclut à la nécessité :

- d'une part, d'organiser dans la métropole, dès que la situation laitiere le permettra, des fabrications de lait en poudre pour les pays d'outre-mer;

- d'autre part, de dresser un plan d'équipement laitier des pays de l'Union française.

A partir des rapports dont nous venons de donner analyse, trois rapports généraux ont été établis :

1. Production rationnelle du lait, par introduction de races appropriées, améliorations des pâturages, etc. Rapporteur : Docteur O.-E. Reed (États-Unis).

2. Production de beurre, fromage et autres dérivés du lait sous les tropiques. Rapporteur : N.-K. Bhargava (Indes).

3. Le ravitaillement des grandes villes tropicales. Rapporteur : E. Lancelot (France).
Ces rapports généraux ne constituent pas de vrais rapports de synthèse et comportent, parfois, des affirmations ou conclusions inexactes ou discutables. Cela tient à ce que les rapports présentés et traitant vraiment de laiterie tropicale étaient à la fois peu nombreux et disparates et aussi, sans doute, au fait que les rapporteurs généraux désignés manquaient de connaissances pratiques suffisantes des pays tropicaux, ou n'avaient qu'une expérience pratique trop fragmentaire de l'élevage et de la production laitière dans les pays sous climat tropical.

Il a été constaté, au cours des séances de travail de la section "Laiterie tropicale », que :

$1^{\circ}$ Les pays tropicaux occupant la plus grande partie de la surface de la terre, il est difficile de classer de manière harmonieuse et utile, dans un seul groupe, "Laiterie tropicale », tout ce qui a trait à la production laitiere dans des pays trop dissemblables, tels le sud des ÉtatsUnis, l'Afrique Occidentale Française, le Kénya, les Indes, car, ce qui peut être vrai dans l'un de ces pays peut être faux pour un autre, du fait, par exemple. de l'altitude et des conditions de vie des autochtones ou du peuplement européen.

Il résulte de celte observation que si la section "Laiterie tropicale " doit se développer dans l'avenir, il y aurait intérêt à la fragmenter en soussections qui pourraient être : Amérique, Arrique, Asie, Océanie.

$2^{\circ}$ Le terme "Laiterie tropicale 》 n'est pas satisfaisant, car il y a lieu d'inclure dans cette section tout ce qui a trait à la production, au traitement et à la distribution du lait dans tous les pays où ces spéculations sont fortement influencées par un climat chaud. Il y aura donc sans doute lieu d'élargir les attributions de la section en changeant sa dénomination.

A la suite des travaux de la Commission de la section "Laiterie tropicale ", le Congrès a adopté les résolutions suivantes:

\section{Résolution I}

Il est désirable que, dans le cadre de la Fédération Internationale de Laiterie, la section "Laiterie tropicale ", créée depuis 1930, soit définitivement organisée et mise en fonctionnement dans le plus bref délai possible.

\section{Résolution II}

Il est souhaitable que la Fédération Internationale de Laiterie entame une action en vue de faciliter, par le moyen d'échanges d'informations et de missions d'etudes, le développement et l'organisation du ravitaillement en lait des villes à population dense des régions tropicales. 